\title{
基于紫外光/臭氧化学改性-选择性化学镀的 聚甲基丙烯酸甲酯表面区域金属化
}

\author{
胡贤巧蒋艳何巧红施积梦胡辰辰汤凝陈恒武* \\ (浙江大学化学系 微分析系统研究所 杭州 310058)
}

\begin{abstract}
摘要 研究以紫外光光化学反应为基础的在聚甲基丙烯酸甲酯(PMMA)表面区域选择性金属化的方法. 通过对无臭氧 紫外光、紫外光十臭氧 $\left(\mathrm{UV} / \mathrm{O}_{3}\right)$ 、臭氧对 PMMA 表面亲水化改性效果的对比, 发现仅有 $\mathrm{UV} / \mathrm{O}_{3}$ 可有效地使 PMMA 表 面亲水. 表面红外光谱表明, $\mathrm{UV} / \mathrm{O}_{3}$ 对 $\mathrm{PMMA}$ 表面协同作用的结果是在辐照区域生成了羧基等含氧活性基团. 以此含 氧活性基团为基础，经过胺化、氯金酸阴离子交换、 $\mathrm{NaBH}_{4}$ 还原等表面反应，在 $\mathrm{UV} / \mathrm{O}_{3}$ 作用区域形成化学镀所必须的 金纳米催化中心, 再将 PMMA 浸入镀金、镀铜等化学镀浴中，即可实现 PMMA 表面的区域金属化. 以打印的菲林片为 原始掩膜，该方法分辨率可达 $50 \mu \mathrm{m}$ 或更小. 以该法制备了金和铜的薄膜微电极、金膜微电热器等金属微器件，并表征 了它们的物理化学性能.
\end{abstract}

关键词 聚甲基丙烯酸甲酯; 选择性金属化; 紫外光; 臭氧; 化学镀

\section{Region-selective Metalization of Poly(methylmethacrylate) Surface Based on UV/ozone Surface Modification}

\author{
$\mathrm{Hu}$, Xianqiao Jiang, Yan He, Qiaohong Shi, Yangmeng \\ $\mathrm{Hu}$, Chenchen Tang, Ning Chen, Hengwu* \\ (Department of Chemistry, Institute of Microanalytical Systems, Zhejiang University, Zijin'gang Campus, \\ Hangzhou 310058)
}

\begin{abstract}
Poly(methylmethacrylate) (PMMA) has been widely used in the fields of optical instruments, aviation and auto industry, construction industry, advertising and decoration industry due to its optical transparency, low cost, low toxicity, and less fragile property. Recently it has been exploited to fabricate microfluidic chips. Region-selective metallization of PMMA surfaces are frequently required to improve the surface properties of PMMA and to integrate micro metal devices in PMMA microfluidic chips. This article presents our recent study on region-selective metalization of PMMA by means of $\mathrm{UV} / \mathrm{O}_{3}$-based surface modification in combination of region-selective electroless plating. Deep UV-lights, ozone, and deep UV-lights in cooperation with ozone were compared with respect to the efficiency of hydrophilization of the PMMA surface. It was observed with water contact angle measurements that only synchronized deep UV and ozone treatment can turn the PMMA surface from hydrophobic to hydrophilic effectively. Attenuated total reflection fourier transform infrared spectra revealed that polar moieties such as carboxyl groups were generated on the $\mathrm{UV} / \mathrm{O}_{3}$ treated areas. These polar moieties served as the anchors for further chemical reactions including amination of the carboxyl groups with ethylenediamine, adsorption of chloraurate anion onto the amine groups, and reduction of the $\mathrm{Au}(\mathrm{III})$ in the chloraurate anion to $\mathrm{Au}(0)$ by borohydride. These reactions led to the formation of the catalysis center of gold nano-particles on the UV-irradiated areas, which is indispensable for region-selective electroless plating. After optimization of the experimental conditions, a novel method for preparation of micro metal devices on PMMA surface has been established. With the printed poly(ethylene terephthalate) (PET) film serving as the primary photo mask, the achievable resolution of the prepared micro metal patterns was $50 \mu \mathrm{m}$ or less. The deposited metal films adhered to the PMMA surface so strongly that they could hardly be tore off with adhesive tapes. Micro gold electrodes, micro gold electric heaters, micro copper electrodes, etc. were demonstrated to be prepared on PMMA surfaces, and their physical and electro-chemical properties were characterized.

Keywords poly(methylmethacrylate); region-selective metalization; $\mathrm{UV} / \mathrm{O}_{3}$; electroless plating
\end{abstract}

\footnotetext{
*E-mail: hwchen@zju.edu.cn; Tel.: +0086-0571-88206773; Fax: +0086-0571-88273572

Received July 25, 2012; published September 21, 2012.

Supporting information for this article is available free of charge via the Internet at http://sioc-journal.cn

Project supported by the National Natural Science Foundation of China (No. 20890020) and Provincial Natural Science Foundation of Zhejiang (No. Y4100254)

项目受国家自然科学基金(No. 20890020)和浙江省自然科学基金(No. Y4100254)资助.
} 


\section{1 引言}

聚甲基丙烯酸甲酯(PMMA)无毒无臭、透光、不易 破碎、价格便宜, 被广泛用于光学仪器、航空与汽车、 建筑、广告和装饰等行业. 由于 PMMA 表面性能稳定, 玻璃化温度较低 $\left(105{ }^{\circ} \mathrm{C}\right)$, 易通过激光刻蚀、热压成型、 热封等工艺进行微细加工, 近年来, 已经被广泛用于制 备微流控芯片 ${ }^{[1-3]}$.

在微流控分析系统中, 经常需要在芯片上集成诸如 电化学检测传感电极、热电偶、电热器等金属微器件. 由 于光胶溶剂会侵蚀高聚物, 因此采用常规的蒸发沉积光刻技术在高聚物芯片上制备金属微器件, 有一定的困 难 $^{[4]}$. 在前期工作中, 我们采用紫外光区域改性结合化 学镀的方法在聚碳酸酯 $(\mathrm{PC})^{[5]}$ 、聚苯乙烯 $(\mathrm{PS})^{[6]}$ 、聚对苯 二甲酸乙二醇酯 $(\mathrm{PET})^{[7]}$ 的微流控电泳芯片上成功制备 了安培检测微电极. 金永龙等 ${ }^{[8]}$ 、唐红肖等 ${ }^{[9]}$ 也采用紫外 光改性结合化学镀法, 分别在聚萗二甲酸乙二醇酯 $(\mathrm{PEN})$ 上制备铜微电极 ${ }^{[8]}$ 和在 $\mathrm{PET}$ 上制备微银薄膜图 形 $[9]$.

本文研究基于紫外光区域改性的选择性化学镀技 术使 PMMA 表面区域金属化的技术. 研究发现，与 PC, PS, PET 等材料不同, PMMA 只有在紫外光和由其产生 的臭氧的协同作用 $\left(\mathrm{UV} / \mathrm{O}_{3}\right)$ 下，其表面才能显著地转为 亲水, 生成为后续的区域化学镀所必须的活性基团. 在 此基础上, 研究建立了以 $\mathrm{UV} / \mathrm{O}_{3}$ 改性-区域化学镀技术 在 PMMA 表面制备金属微器件的方法.

\section{2 结果与讨论}

\subsection{PMMA 表面活化改性}

采用紫外光区域改性结合化学镀技术在高聚物表 面局部区域化学镀金属薄膜, 最为关键的是对拟镀的高 聚物表面进行区域活化(紫外光照)处理, 在该区域形成 羧基等含氧亲水性活性基团, 并通过后续的一系列化学 反应，在其表面形成纳米金催化中心 ${ }^{[10]}$, 浸入镀浴后， 镀浴中的金属离子在纳米金的催化下，还原成金属原子 并定向沉积在拟镀区域, 形成金属薄膜. 整个过程的化 学反应可参见支持信息 1 .

然而，当我们采用文献[6]所报道的无臭氧低压录 灯(简写为 UV-254 灯, 主要辐射 $254 \mathrm{~nm}$ 线) 和类似的方 法在 PMMA 表面进行区域改性和化学镀金时发现, 在 PMMA 表面不能形成牢固的、具有光泽的金镀层. 水接 触角测量数据表明, 在 UV-254 灯辐射 $2 \mathrm{~h}$ 后(254 nm 的 辐照剂量为 $36 \mathrm{~J} / \mathrm{cm}^{2}$ ), 接触角仅仅从 $79^{\circ}$ 下降到约 $61^{\circ}$, 见图 1a. 继续增长辐照时间, 接触角变化很小. 这表明 UV-254 灯的辐照不能显著地改善 PMMA 表面的亲水 性. 表面红外实验表明, UV-254 灯辐射前后, PMMA 的 表面红外谱图除部分峰减弱外并未有新的峰产生(图 2), 即 UV-254 灯辐射并未在 PMMA 表面形成羧基等活性
基才，因而也就不能通过后续的胺化、离子交换、还原 等步骤在光照区域形成化学镀所必须的纳米金催化中 心.

有文献报道 ${ }^{[11]}$, 采用紫外光辐照结合由深紫外光 诱导产生的 $\mathrm{O}_{3}$ 的作用, 可显著改善 PMMA 表面的亲水 性. 于是, 我们采用 ZF-1 型三用紫外线分析仪中能产 生臭氧的低压录灯(简写为 $U V / O_{3}$ 灯)对 PMMA 表面进 行光化学改性处理, 效果如图 $1 \mathrm{~b}$ 所示: PMMA 表面水的 接触角随光照时间的增加而迅速降低，当光照时间达到 $2 \mathrm{~h}$ 后，接触角已经减小到 $21^{\circ}$. 低压求灯的主射线为 $254 \mathrm{~nm}$ 紫外光, 但也辐射 $185 \mathrm{~nm}$ 紫外光, 当其穿过石 英灯管进入空气后，能被空气中的氧气吸收后产生臭 氧 ${ }^{[11,12]}$. 而无臭氧的 UV-254 灯由于在石英灯管中加入 了足够数量的钛元素，使得 $200 \mathrm{~nm}$ 以下的紫外线截止， 所以 UV-254灯的主要射线为 $254 \mathrm{~nm}$, 也不会产生臭氧. 由于 $\mathrm{UV} / \mathrm{O}_{3}$ 灯除辐射 254 和 $185 \mathrm{~nm}$ 紫外光外, 还有臭 氧产生, 为了检验是否由于臭氧的作用而导致 PMMA 表面亲水化, 我们将 $\mathrm{UV} / \mathrm{O}_{3}$ 灯的紫外光遮挡后, 仅将 PMMA 表面暴露在紫外光所产生的 $\mathrm{O}_{3}$ 气氛中. 实验结 果显示, 仅有 $\mathrm{O}_{3}$ 则完全不能使 PMMA 表面亲水化(图 1c). 因此, $\mathrm{UV} / \mathrm{O}_{3}$ 对 PMMA 表面的改性是紫外光和 $\mathrm{O}_{3}$ 的协同作用. 通过比较 PMMA 表面红外图谱(图 2)可以 发现, $\mathrm{UV} / \mathrm{O}_{3}$ 处理后 $1725 \mathrm{~cm}^{-1}$ 处的主羰基峰减弱, 而主 羰基峰两侧的 $1800 \sim 1750$ 和 1715 1670 $\mathrm{cm}^{-1}$ 的峰有所 增强, 可以归属为 $\mathrm{UV} / \mathrm{O}_{3}$ 处理所产生的新羰基峰与原羰 基峰的边缘叠加引起; 伴随着新羰基峰的产生, 在 $3650 \sim 3100 \mathrm{~cm}^{-1}$ 也出现比较明显的宽峰, 可以归属为 $\mathrm{O}-\mathrm{H}$ 的伸缩振动, 表明了有一 $\mathrm{OH}$ 产生; $2990 \mathrm{~cm}^{-1}$ 和 $2950 \mathrm{~cm}^{-1}$ 处的甲基和亚甲基的 $\mathrm{C}-\mathrm{H}$ 伸缩振动峰有所 减弱，表明部分甲基亚甲基断裂或者被氧化. 据此我们

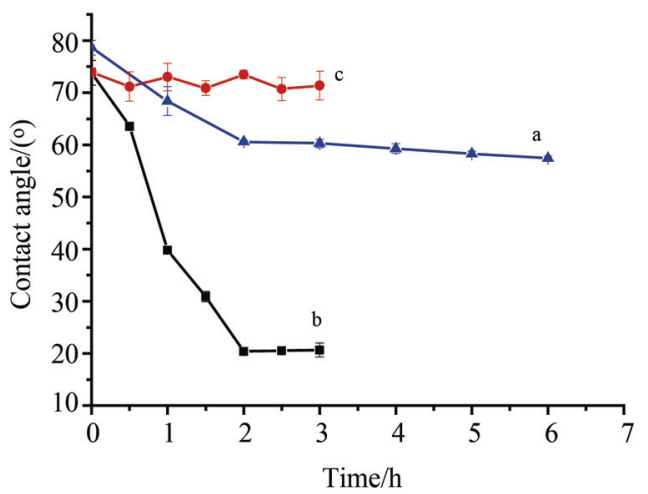

图 1 PMMA 表面测得的水接触角与表面处理时间的关系 (a)无臭氧的低压录灯辐照, $254 \mathrm{~nm}$ 的辐射强度为 $5.08 \mathrm{~mW} / \mathrm{cm}^{2}$; (b) 能产生 臭氧的低压录灯 $\left(\mathrm{UV} / \mathrm{O}_{3}\right.$ ) 辐照, $254 \mathrm{~nm}$ 的辐射强度为 $2.10 \mathrm{~mW} / \mathrm{cm}^{2}$; (c) 仅用 (b)灯所产生的臭氧处理

Figure 1 Water contact angles of PMMA surfaces after treated with different sources for varied times

(a) irradiated with $\mathrm{O}_{3}$-free low pressure mercury lamp (the irradiation power was measured $5.08 \mathrm{~mW} / \mathrm{cm}^{2}$ at $254 \mathrm{~nm}$ ); (b) irradiated with $\mathrm{O}_{3}$-generating low pressure mercury lamp (the irradiation power was measured $2.10 \mathrm{~mW} / \mathrm{cm}^{2}$ at $254 \mathrm{~nm}$ ); (c) treated only with $\mathrm{O}_{3}$ generated by the source used in (b) 


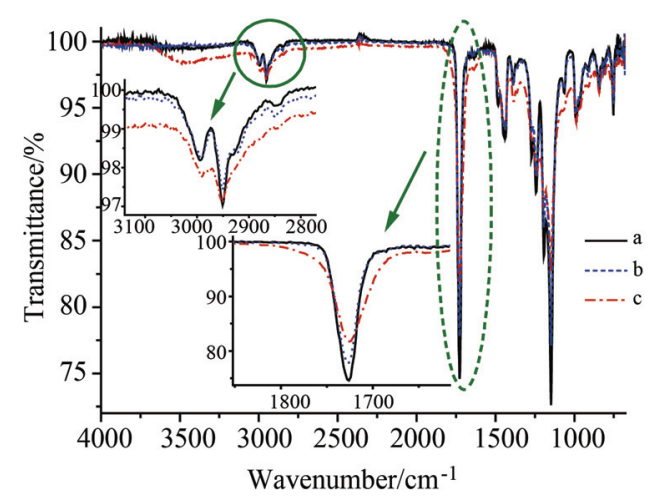

图 2 紫外灯表面处理前后的 PMMA 表面衰减全反射红外图谱 (a)原始 PMMA; (b)经无臭氧低压永灯辐照后的 PMMA; (c)经能产生臭氧 的低压录灯辐照后的 PMMA

Figure 2 Attenuated total reflection Fourier transform infrared spectra of PMMA sheets before and after irradiated with UV lamps

(a) native PMMA, (b) PMMA irradiated with the $\mathrm{O}_{3}$-free low pressure mercury lamp; (c) PMMA irradiated with the $\mathrm{O}_{3}$-generating low pressure mercury lamp

认为, 在 $\mathrm{UV} / \mathrm{O}_{3}$ 处理过程中, 254 和 $185 \mathrm{~nm}$ 紫外光具有 很强的能量, 可被 PMMA表面吸收, 从而使主链或者侧 链甲基断裂并形成自由基 ${ }^{[13,14]}$; 另外, 产生的臭氧进一 步吸收 $254 \mathrm{~nm}$ 紫外光而解离产生氧自由基, 产生的氧 自由基与 PMMA 表面的自由基进行反应, 从而在 PMMA 表面引入含氧的羰基、羧基、羟基等极性基团 ${ }^{[13]}$, 使 PMMA 表面亲水性增强. 所以, 对 PMMA 表面的紫 外光化学改性, 需要紫外光和臭氧协同作用.

根据以上实验, 在后续的试验中均采用 $\mathrm{UV} / \mathrm{O}_{3}$ 作为 光源, 通过掩膜对 PMMA进行区域改性. 在使用掩膜的 情况下, 掩膜与 PMMA 片之间残留空气是形成 $\mathrm{O}_{3}$ 的保 障.

\section{2 形成金纳米催化中心的实验条件优化}

将经过光化学改性的 PMMA 片浸入到含有偶合剂 $\mathrm{EDC}$ 的乙二胺溶液进行胺化处理, PMMA 表面生成的羧 基与乙二胺的一个氨基反应生成酰胺, 而另一个氨基则 暴露在 PMMA 表面, 可以通过离子交换作用与 $\mathrm{HAuCl}_{4}$ 溶液中的 $\mathrm{AuCl}_{4}^{-}$结合, 经 $\mathrm{NaBH}_{4}$ 溶液处理后, 在紫外改 性区域形成一层有催化活性的 $\mathrm{Au}$ 纳米粒子(可参见支持 信息 1).

在文献[6]中, 胺化处理需要 $3 \mathrm{~h}, \mathrm{HAuCl}_{4}$ 处理时间 需要 $2.5 \mathrm{~h}$, 耗费的时间较长. 本文对这两步的处理时间 作进一步的优化. 结果表明胺化时间从 $3 \mathrm{~h}$ 缩短到 $2 \mathrm{~h}$, 不影响胺化效果. 图 3 是用 $\mathrm{UV} / \mathrm{O}_{3}$ 通过条纹状掩膜光照 处理、胺化后的 PMMA 表面, 再与苂光试剂异硫氰酸荧 光素(FITC)反应后所得到的荧光显微图像, 其中亮条纹 (曝光部分)与 $\mathrm{UV} / \mathrm{O}_{3}$ 改性时所用的掩膜图形一致, 表明 在 PMMA 表面由 $\mathrm{UV} / \mathrm{O}_{3}$ 作用所生成的羒基已成功地实 现胺化. 另外, 将 $\mathrm{HAuCl}_{4}$ 处理时间从文献[6]的 $2.5 \mathrm{~h}$ 缩 短为 $30 \mathrm{~min}$, 即能得到良好的化学镀金膜.

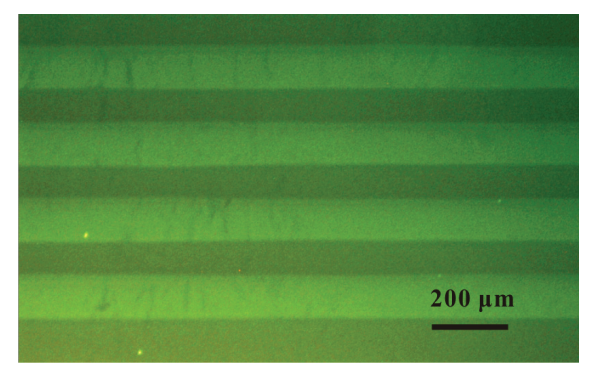

图 $3 \mathrm{UV} / \mathrm{O}_{3}$ 图案化 $\mathrm{PMMA}$ 表面的苂光显微 $\mathrm{CCD}$ 图像

PMMA 片用 $\mathrm{UV} / \mathrm{O}_{3}$ 灯经直条纹状掩膜光照 $2 \mathrm{~h}$ 、胺化 $2 \mathrm{~h}$ 后, 浸入到 FITC 的嗍砂溶液 $(\mathrm{pH}=9)$ 中反应 $20 \mathrm{~h}$. 荧光 $\mathrm{CCD}$ 图像由配置有 $\mathrm{CCD}$ 相机的倒 置荧光显微镜摄取

Figure 3 Fluorescence $\mathrm{CCD}$ image of $\mathrm{UV} / \mathrm{O}_{3}$ patterned and aminated PMMA surface

A PMMA sheet was subjected to $2 \mathrm{~h} \mathrm{UV/O}{ }_{3}$ treatment through a photomask with straight lines, followed by amination with ethylenediamine for $2 \mathrm{~h}$. Afterwards, the aminated PMMA was immersed into a FITC solution buffered with sodium tetraborate $(\mathrm{pH}=9)$ for $20 \mathrm{~h}$. The fluorescence image was taken with an inversed fluorescence microscopy equipped with a CCD camera

\subsection{PMMA 上化学镀金膜的性能}

按 4.1 节的方法，在 PMMA 表面化学镀金所得到的 金膜微电极阵列见图 4. 为了测试该方法制备的金膜微 器件的附着牢度, 在 PMMA 片上用上述方法镀上 $23 \times$ 29 个直径为 $0.5 \mathrm{~mm}$ 的金膜点阵, 用 $3 \mathrm{M}$ 胶带纸 $(3 \mathrm{M}$ 公 司, 美国)试验测试其附着牢度 ${ }^{[15]}$. 结果表明, 刚镀好的 金膜附着力欠强, 只有 $85 \%$ 的金膜点阵能够通过胶带纸 试验(不为胶带纸所剥离, 图 5a). 在 $90{ }^{\circ} \mathrm{C}$ 的烘箱中褪 火 $3 \mathrm{~h}$ 后, 99\%以上的点阵可通过胶带纸试验(图 $5 \mathrm{~b}$ ). (a)

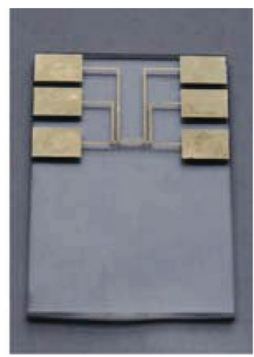

(c)

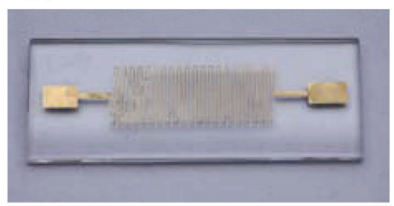

(b)

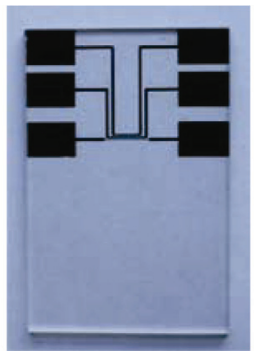

(d)

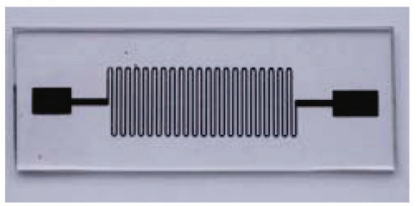

图 4 所制备的金膜微电极阵列 $(a, b)$ 和金膜微电热器 $(c, d)$ 的照片 (a)和(b)中三个金电极的传感部分宽度分别为 $100,250,250 \mu \mathrm{m}$, 间隔 250 $\mu \mathrm{m}$; (c)和 $(\mathrm{d})$ 电热器金膜线的宽度为 $200 \mu \mathrm{m}$, 间隔 $500 \mu \mathrm{m}$. (a) 和(c), 从正 面看; (b)和(d), 从反面看

Figure 4 Photo pictures of micro gold film electrode array $(a, b)$ and of an electric heater $(c, d)$ prepared on PMMA sheets with the developed method

The widths of the sensing parts of the three micro gold electrodes in (a) and (b) are $100,250,250 \mu \mathrm{m}$, respectively, the between-spacing is $250 \mu \mathrm{m}$. The line-width of the micro gold electric heater in (c) and (d) is $200 \mu \mathrm{m}$, and the between-spacing is $500 \mu \mathrm{m}$. (a) and (c), front-side view, (b) and (d) back-side view 
(a)

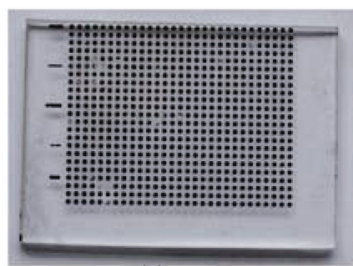

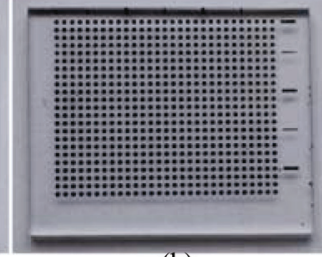

(b)
图 5 PMMA 上化学镀金点阵的附着牢度试验结果

退火前(a)和退火后(b)的 $23 \times 29$ 个化学镀金点阵经胶带纸粘合-剥离试验 后的情况. 退火条件, $90{ }^{\circ} \mathrm{C} 3 \mathrm{~h}$

Figure 5 The electrolessly plated gold dots remained on PMMA surface after the tape-test

Totally $23 \times 29$ dots were subjected to the tape-test (a) before and (b) after annealing at $90{ }^{\circ} \mathrm{C}$ for $3 \mathrm{~h}$

用循环伏安法表征金膜微电极的电化学性质, 并且 与标准金圆盘电极的电化学性质进行对比. 结果(图 6) 表明, PMMA 片上金膜微电极的电化学性质与金圆盘电 极非常相似: 两者的循环伏安图中的氧化峰电位、还原 峰电位以及氧化还原峰电位之差都很相近; 同批制备的 金膜电极片, 峰电位完全一致, 氧化峰峰值电流的 RSD 为 $3.8 \%(n=3)$. 表明该法制作的金膜微电极的电化学 性能良好，且方法具有较好的重现性.

采用该方法制备了微电热器, 其欧姆曲线和电压温度曲线见图 7. 随着所加电压的增加, 温度大致成指 数上升, 符合焦耳-楞次定律. 根据欧姆曲线的斜率, 得 到图 $4 \mathrm{c}$ 加热器的电阻为 $2.8 \mathrm{k} \Omega$. 通过改变施加的电压 和加热器的电阻(即金膜导线的长度)可以调节加热温度 和加热区域, 以满足不同的需要.

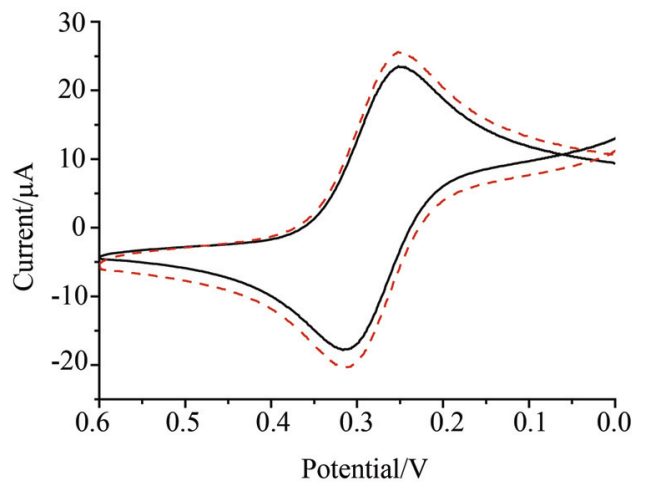

图 6 标准金圆盘电极(黑实线)和 PMMA 片上的金膜微电极(红虚线) 的循环伏安图

电解质溶液: $5 \mathrm{mmol} / \mathrm{L} \mathrm{K}{ }_{3} \mathrm{Fe}(\mathrm{CN})_{6}+1 \mathrm{~mol} / \mathrm{L} \mathrm{KCl}$ 溶液; 扫描速度: $50 \mathrm{mV} / \mathrm{s}$; 响应面积: 标准金电极 $3.14 \mathrm{~mm}^{2}$, 金膜微电极 $3.24 \mathrm{~mm}^{2}$. 循环伏安实验采 用了三电极体系, 金膜微电极或金圆盘电极为工作电极, $\mathrm{Ag} / \mathrm{AgCl}$ 为参比 电极, 铂丝为对电极

Figure 6 Cyclic voltammetry graphs obtained with a commercial micro gold disk electrode (black solid line) and a micro gold film electrode prepared on a PMMA sheet (red dash line)

Electrolyte solution, $5 \mathrm{mmol} / \mathrm{L} \mathrm{K} 33 \mathrm{Fe}(\mathrm{CN})_{6}$ prepared in $1 \mathrm{~mol} / \mathrm{L} \mathrm{KCl}$ solution; scanning rate, $50 \mathrm{mV} / \mathrm{s}$; the exposure area of the electrodes, $3.14 \mathrm{~mm}^{2}$ for micro gold disk electrode and $3.24 \mathrm{~mm}^{2}$ for micro electrolessly plated gold film electrode; Three electrode systems, either a micro gold disk electrode or a micro electroless gold film electrode cooperated with a $\mathrm{Ag} / \mathrm{AgCl}$ wire reference electrode and a platinum wire counter electrode

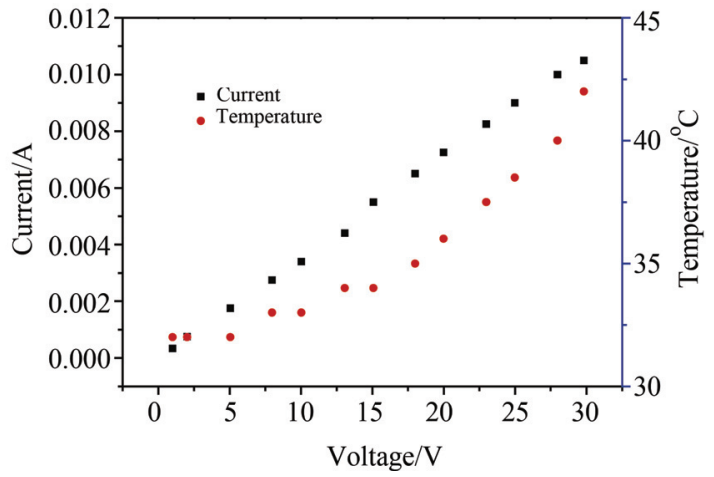

图 7 所制备的金微加热器的欧姆曲线和电压-温度曲线 $30 \mathrm{~V}$ 为所用直流电源的最高输出电压

Figure 7 An Ohm curve and a voltage-temperature curve obtained by application of varied voltages to the micro film gold electric heater prepared on a PMMA sheet

$30 \mathrm{~V}$ was the highest output of the employed DC voltage supplier

\subsection{PMMA 上化学镀铜}

按 4.2 节的方法在 PMMA 上区域化学镀铜所得到的 图案和微电极阵列见图 8.

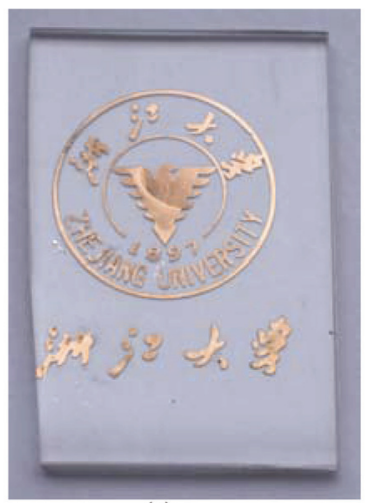

(a)

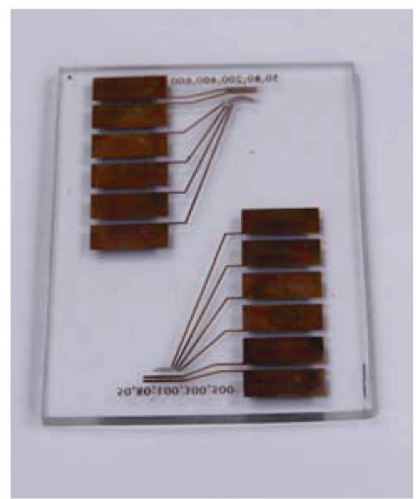

(b)
图 8 所制备的铜膜校徽图形(a)和铜膜微电极阵列(b)的照片 (b)中铜电极传感部分的宽度分别为以电极附近的镀铜数字标注; (a)从铜 膜背面看, (b)从铜膜正面看

Figure 8 Photo pictures of a copper-film school-badge pattern (a) and a micro copper film electrode array (b) prepared with the developed method The widths of the sensing parts of the micro copper electrodes in (b) are plated out with the numbers located near the electrodes. (a) back-side view, (b) front-side view

\section{3 结论}

本文研究表明, 分别采用深紫外光或 $\mathrm{O}_{3}$ 对 PMMA 表面进行改性, 并不能有效地在 PMMA 表面形成亲水 的羟基羧基等活性基团; 只有深紫外光与由其在空气中 所生成的 $\mathrm{O}_{3}$ 协同作用 $\left(\mathrm{UV} / \mathrm{O}_{3}\right)$ 下, 才能使 PMMA 表面产 生羟基羧基等活性基团. 采用 $\mathrm{UV} / \mathrm{O}_{3}$ 作为表面活化改性 方法, 对 PMMA 进行了选择性区域改性, 然后经过胺 化、 $\mathrm{HAuCl}_{4}$ 离子交换、 $\mathrm{NaBH}_{4}$ 还原等步骤，可在紫外光 照后 PMMA 的表面形成区域选择性化学镀所需的纳米 金催化中心, 浸入适当的化学镀浴中即可在光照区选择 性沉积金、铜等金属薄膜. 用这种选择性化学镀制备的 
金膜和铜膜微器件尺寸精确(受菲林掩膜打印精度的限 制, 分辨率可达 $50 \mu \mathrm{m}$ 或更小), 电学和电化学性能良 好, 有较强的附着强度. 使用该法在 PMMA上制备金属 微薄膜器件无需真空蒸发仪或磁控浌射机等特殊设备, 也无须考虑光胶及溶剂对其的腐蚀作用, 简单易行, 在 普通实验室即可实现. 通过更改化学镀浴, 本法也可拓 展到在 PMMA 表面区域选择性化学镀 $\mathrm{Ni}, \mathrm{Ag}, \mathrm{Pt}$ 等金属 薄膜.

\section{4 实验部分}

\section{1 金膜微器件}

PMMA 上制作金属器件的步骤如图 9 所示.

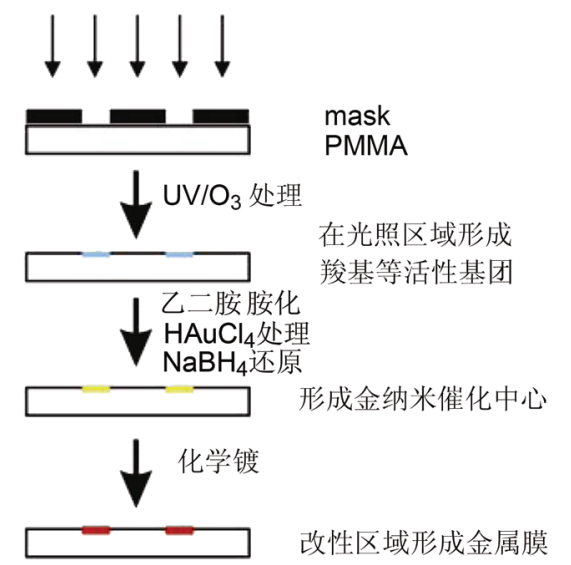

图 9 PMMA 上金属器件的制作步骤

Figure 9 The procedure for preparing micro metal devices on PMMA surface

取 $4 \mathrm{~cm} \times 6 \mathrm{~cm}$ 的 PMMA 一片, 将其与石英掩膜 ${ }^{[16]}$ 贴合, 夹紧后置于 ZF-1 型三用紫外线分析仪(移去灯罩 下的滤光板)的紫外灯管下，使其与灯管的距离约为 3 $\mathrm{cm}\left(254 \mathrm{~nm}\right.$ 的辐照强度为 $\left.2.10 \mathrm{~mW} / \mathrm{cm}^{2}\right)$, 辐照 $2 \mathrm{~h}$ 后取 出. 将光照后的 PMMA 片浸入到 $0.36 \mathrm{~mol} / \mathrm{L}$ 的乙二胺溶 液中(用 $\mathrm{pH}=7.0$ 的 $100 \mathrm{mmol} / \mathrm{L}$ 磷酸缓冲液配制, 含 50 $\mathrm{mmol} / \mathrm{L}$ 的 $\mathrm{EDC})$, 室温下反应 $2 \mathrm{~h}$. 取出后用水冲洗, 吹 干, 浸入到 $1 \mathrm{mmol} / \mathrm{L}$ 的氯金酸溶液中反应 $30 \mathrm{~min}$. 水洗 后, 用 $0.1 \mathrm{~mol} / \mathrm{L}$ 的硼氢化钠溶液处理 $10 \mathrm{~min}$. 取出, 水 洗, 吹干. 为了消除非光照区由于非选择性吸附所引起 的过镀现象, 在化学镀之前, 把 PMMA 片浸到 0.5 $\mathrm{mol} / \mathrm{L}$ 的硫氰酸钾溶液中, 超声清洗 $10 \mathrm{~min}$. 最后将活 化后的 PMMA 浸入到化学镀金液(含有 $0.125 \mathrm{~mol} / \mathrm{L}$ 亚 硫酸钠, $0.6 \mathrm{~mol} / \mathrm{L}$ 甲醛和 $8 \mathrm{mmol} / \mathrm{L}$ 的亚硫酸金钠溶液) 中，室温反应 $40 \mathrm{~min}, \mathrm{PMMA}$ 表面光照区形成光亮的金 膜. 将镀有金膜的 PMMA片置于两片厚玻璃之间，放入 干燥箱中, 升温至 $90{ }^{\circ} \mathrm{C}$, 保持 $3 \mathrm{~h}$ 后, 降至室温, 取出 片子.

\section{2 铜膜微器件}

紫外光照和活化处理同 4.1 节. 经 $0.5 \mathrm{~mol} / \mathrm{L}$ 的硫氰 酸钾溶液超声清洗 $10 \mathrm{~min}$ 后，将活化后的 PMMA 浸入 到化学镀铜液 $(0.5 \mathrm{~g}$ 五水硫酸铜、 $2.5 \mathrm{~g}$ 酒石酸钾钠, $0.5 \mathrm{~g}$ 氢氧化钠、 $1 \mathrm{~mL}$ 甲醛配于 $100 \mathrm{~mL}$ 去离子水中)中, 室温 反应 $90 \mathrm{~min}$ 左右, 在 PMMA 的光照区可得到光亮的紫 红色铜膜.

\section{References}

[1] Chen, Y.; Zhang, L. Y.; Chen, G. Electrophoresis 2008, 29, 1801.

[2] Jubery, T. Z.; Hossan, M. R.; Bottenus, D. R.; Ivory, C. F.; Dong, W. J.; Dutta, P. Biomicrofluidics 2012, 6, 016503.

[3] Henry, A. C.; Tutt, T. J.; Galloway, M.; Davidson, Y. Y.; McWhorter, C. S.; Soper, S. A.; McCarley, R. L. Anal. Chem. 2000, 72,5331 .

[4] Liu, J. S.; Luo, Y.; Du, Y.; Liu, C.; Wang, L. D.; Zhou, W. H.; Yang, X. R. Chin. J. Anal. Chem. 2005, 33, 584. (刘军山，罗怡，杜 艳, 刘冲, 王立鼎, 周伟红, 杨秀荣, 分析化学, 2005, 33, 584.)

[5] Kong, Y.; Chen, H. W.; Wang, Y. R.; Soper, S. A. Electrophoresis 2006, 27, 2940

[6] Hu, X. Q.; He, Q. H.; Lu, H.; Chen, H. W. J. Electroanal. Chem. 2010, 638, 21.

[7] Hao, Z. X.; Chen, H. W.; Zhu, X. Y.; Li, J. M.; Liu, C. J. Chromatogr. A 2008, 1209, 246.

[8] Jin, Y. L.; Zhang, Y.; Gu, N. Chem. J. Chin. Univ. 2008, 29, 1859. (金永龙, 张宇, 顾宁, 高等学校化学学报, 2008, 29, 1859.)

[9] Tang, H. X.; Wu, Z. K.; Yang, J.; Li, S. Y. China Surface Engineering 2012, 25, 99. (唐红肖，吴仲崩，杨军，李少英，中国表面工程， 2012, 25, 99.)

[10] Kong, Y. Ph.D. Dissertation, Zhejiang University, Hangzhou, 2006. (孔泳, 博士论文, 浙江大学, 杭州, 2006.)

[11] Tsao, C. W.; Hromada, L.; Liu, J.; Kumar, P.; DeVoe, D. L. Lab Chip 2007, 7, 499.

[12] Vig, J. R. J. Vac. Sci. Technol. A 1985 3, 1027.

[13] Fan, Y. Q.; Liu, Y.; Li, H. W.; Foulds, I. G. J. Micromech. Microeng. 2012, 22, 027001.

[14] Hozumi, A.; Inagaki, H.; Kameyama, T. J. Colloid Interface Sci. 2004, 278, 383

[15] Baker, L. A.; Zamborini, F. P.; Sun, L.; Crooks, R. M. Anal. Chem. 1999, $71,4403$.

[16] Kong, Y.; Chen, H. W.; Yun, X.; Hao, Z. X.; Fang, Z. L. Chin. J. Anal. Chem. 2007, 35, 623. (孔泳, 陈恒武, 云晓, 郝振霞, 方肇 伦, 分析化学, 2007, 35, 623.) 